\title{
A new double-tracer gas single-breath washout to assess early cystic fibrosis lung disease
}

\author{
Florian Singer*, Georgette Stern*, Cindy Thamrin*,", Chiara Abbas*, \\ Carmen Casaulta*, Urs Frey* ${ }^{\star \star}$ and Philipp Latzin*
}

ABSTRACT: In cystic fibrosis (CF), tests for ventilation inhomogeneity are sensitive but not established for clinical routine. We assessed feasibility of a new double-tracer gas single-breath washout (SBW) in school-aged children with CF and control subjects, and compared SBW between groups and with multiple-breath nitrogen washout (MBNW).

Three SBW and MBNW were performed in 118 children (66 with CF) using a side-stream ultrasonic flowmeter setup. The double-tracer gas containing $5 \%$ sulfur hexafluoride and $26.3 \%$ helium was applied during one tidal breath. Outcomes were SBW phase III slope (SIIIDTG), MBNWderived lung clearance index $(\mathrm{LCl})$, and indices of acinar (Sacin) and conductive (Scond) ventilation inhomogeneity.

SBW took significantly less time to perform than MBNW. SBW and MBNW were feasible in 109 (92.4\%) and 98 (83.0\%) children, respectively. SIIIDTG differed between children with CF and controls, mean $\pm \mathrm{SD}$ was $-456.7 \pm 492.8$ and $-88.4 \pm 129.1 \mathrm{mg} \cdot \mathrm{mol} \cdot \mathrm{L}^{-1}$, respectively. Abnormal SIIIDTG was present in $36(59 \%)$ children with CF. SIIIDTG was associated with LCl $(r=-0.58)$ and Sacin $(r=-0.58)$, but not with Scond.

In CF, steeply sloping SIIIDTG potentially reflects ventilation inhomogeneity near the acinus entrance. This tidal SBW is a promising test to assess ventilation inhomogeneity in an easy and fast way.

KEYWORDS: Children, efficiency, helium, respiratory function tests, small airway remodelling, sulfur hexafluoride

onventional measurements of airways obstruction are inadequate to characterise early cystic fibrosis (CF) lung disease. Compared with spirometry, the lung clearance index (LCI), a measure of overall ventilation inhomogeneity derived from inert tracer gas multiple-breath washout (MBW), is more sensitive for peripheral structural airway pathology detected by computer tomography [1-4]. LCI is associated with peripheral airways infection and seems to be a relevant end-point in clinical CF trials [5-8]. Despite this, MBW has not been applied to routine clinical care due to the long duration and the need for unavailable or customised equipment, such as mass spectrometer and photo-acoustic setups $[3,9]$. Alternatively, tidal phase III slope (SIII) analyses from tracer gas single-breath washout (SBW) tests provide insight into overall and regional ventilation inhomogeneity [10-13].
Simultaneous use of different tracer gases, e.g. helium $(\mathrm{He})$ and sulfur hexafluoride $\left(\mathrm{SF}_{6}\right)$, potentially yields information regarding the site at which ventilation inhomogeneity arises [10, 14]. However, SBW is also not routinely established as most SBW procedures require vital capacity (VC SBW) manoeuvres at pre-set flow rates, and also rely on mass spectrometers [12, 15].

A more practical lung function test for ventilation inhomogeneity based on available equipment could overcome previous drawbacks. We recently developed a new and easy SBW test requiring tidal breathing only (tidal SBW) and involving a double-tracer gas mixture containing $\mathrm{He}$ and $\mathrm{SF}_{6}$ to assess regional ventilation inhomogeneity in adults [16]. We were able to show that the ultrasonic flowmeter setup can accurately trace $\mathrm{He}$ and $\mathrm{SF}_{6}$ washout as validated against mass spectrometry [16].

\section{AFFILIATIONS}

${ }^{*}$ Division of Respiratory Medicine, Dept of Paediatrics, University Hospital of Bern, Bern, and 'University Children's Hospital Basel, Switzerland.

${ }^{*}$ Woolcock Institute of Medical Research, Glebe, NSW, Australia.

CORRESPONDENCE

P. Latzin

Division of Respiratory Medicine, Dept of Paediatrics

University Children's Hospital of Bern Freiburgstrasse

3010 Bern

Switzerland

E-mail: philipp.latzin@insel.ch

Received:

March 132012

Accepted after revision:

May 052012

First published online:

May 172012 
In the present study, we determined whether the double-tracer gas SBW-derived tidal phase III slope (SIIIDTG) detects early lung disease in school-aged children with CF. Study outcomes were: 1) feasibility, 2) ability to detect increased ventilation inhomogeneity in CF, and 3) the association of SIIIDTG with indices from multiple-breath nitrogen washout (MBNW) and spirometry.

\section{METHODS}

\section{Study participants}

We prospectively enrolled 121 children aged between 5 and 16 yrs at the outpatient clinics, Children's University Hospital Bern, Bern, Switzerland, as detailed in the online supplementary material. Tidal SBW and MBNW were performed in triplicate, with children with $\mathrm{CF}$ additionally performing spirometry [17] in that order. The study was approved by the Ethics Committee of the Canton of Bern, Bern, Switzerland. The children's consent was obtained and parents or caregivers provided written informed consent for this study.

\section{Double-tracer gas SBW}

We used an available setup (Exhalyzer D; Eco Medics AG, Duernten, Switzerland) described in the online supplementary material. The double-tracer gas mixture contained $26.3 \% \mathrm{He}$, $5 \% \mathrm{SF}_{6}, 21 \%$ oxygen, and balance nitrogen (Carbagas, Bern, Switzerland). This double-tracer gas mixture has the same molar mass as air, such that any detectable changes compared with normally expired molar mass can be attributed to relative changes in $\mathrm{He}$ and $\mathrm{SF}_{6}$ concentrations [16]. Molar mass is measured by a side-stream ultrasonic flowmeter, tidal flows by a mainstream ultrasonic flowmeter.

During normal breathing, the double-tracer gas was applied for a single tidal inhalation prior to exhalation to functional residual capacity [16]. We used Matlab (R2006a; The Mathworks Inc., Natick, MA, USA) for signal processing and analyses. The tidal SBW-derived molar mass expirogram was plotted against expired volume. Two blinded investigators applied a linear fit to the tidal phase III between $65 \%$ and $95 \%$ of expired volume. The primary outcome was the mean SIIIDTG of three technically acceptable tidal SBW. Further details on tidal SBW measurement and analysis are given in the online supplementary material.

\section{Multiple-breath nitrogen washout}

The reference lung function test was MBNW performed using validated equipment [18], according to guidelines [17] and is detailed in the online supplementary material. Primary outcome was LCI calculated as the ratio of cumulative expired volume divided by functional residual capacity $[19,20]$. Secondary outcomes were indices of conductive (Scond) and acinar (Sacin) ventilation inhomogeneity, with their values increasing with increasing ventilation inhomogeneity [21]. LCI is thought to reflect global ventilation inhomogeneity whereas Sacin, derived from the first nitrogen SIII, reflects regional acinar ventilation inhomogeneity. Scond, calculated from the slope across multiple nitrogen SIII over lung turnovers of 1.5 to 6 reflects regional conductive ventilation inhomogeneity [21].

\section{Statistics}

We computed success rates and duration for tidal SBW and MBNW test sessions, and compared lung function data between groups using unpaired t-tests. z-scores for SIIIDTG, LCI, Sacin and Scond were calculated from the healthy study population for forced expiratory volume in $1 \mathrm{~s}$ (FEV1) and forced expiratory flow at $25-75 \%$ of forced vital capacity (FEF25$75 \%$ ) from reference data [22]. We assessed the association of SIIIDTG with these lung function outcomes using Pearson's correlation and applied Bonferroni's correction. We also normalised SIIIDTG by multiplying it by the tidal volume $(V \mathrm{~T})$ of the corresponding breath (SnIIIDTG) and assessed intra-test variability by the coefficient of variation (CV) of SIIIDTG and SnIIIDTG. Analyses quality, i.e. linear regression $\mathrm{R}^{2}$ of SIIIDTG fitting, and SIIIDTG agreement between observers were determined by paired t-tests and intra-class correlation coefficient, respectively. A p-value $<0.05$ was considered statistically significant, and analyses were performed using STATA (release 11; StataCorp, College Station, TX, USA).

\section{RESULTS}

\section{Success rate and duration}

Out of 121 children enrolled, 118 children (66 with CF and 52 controls) performed triple tidal SBW tests (fig. 1). Clinical and demographic characteristics of the study population are presented in table 1. SIIIDTG success rate was high and similar between groups: 109 (92.4\%) children (61 children with CF) achieved 318 acceptable tidal SBW measurements. Six (5\%) children were aged $<6$ yrs and achieved tidal SBW measurements only. Reasons for single test rejection were unsteady breathing, e.g. inspiratory flow exceeding bias flow $(n=22,6 \%)$, lack of phase III $(n=12,3 \%)$ and technical errors $(n=2,1 \%)$. An example of typical double-tracer gas tidal SBW expirograms is given in figure 2. MBNW measurements were achieved by 54 (82\%) children with $\mathrm{CF}$ and 44 (85\%) controls. The mean (range) duration for testing and analyses of three tidal SBW trials was $3.3(2.5-4.1) \mathrm{min}$ and 29.9 (13.9-44.0) $\mathrm{min}$ for three MBNW trials $(\mathrm{p}<0.001)$.

\section{Double-tracer gas phase III slope in children with CF}

Compared with control subjects, children with CF had clearly different double-tracer gas washout patterns during phase III of the tidal SBW (table 1 and fig. 2). In children with $\mathrm{CF}$, SIIIDTG mean $\pm \mathrm{SD}$ was $-456.7 \pm 492.8 \mathrm{mg} \cdot \mathrm{mol} \cdot \mathrm{L}^{-1}$ compared with $-88.4 \pm 129.1 \mathrm{mg} \cdot \mathrm{mol} \mathrm{L}^{-1}$ in healthy children. Mean difference $(95 \% \mathrm{CI})$ of SIIIDTG between children with CF and control subjects was $368.3(223.4-513.2) \mathrm{mg} \cdot \mathrm{mol} \cdot \mathrm{L}^{-1}(\mathrm{p}<0.001)$. SnIIIDTG similarly differed between children with CF and controls. Mean difference $(95 \% \mathrm{CI})$ was $124.1(68.9-179.4) \mathrm{mg} \cdot \mathrm{mol}^{-1}(\mathrm{p}<0.001)$. Additional results are given in the online supplementary material.

In our CF population, sensitivity and specificity for abnormal lung function (>2 z-scores) was 59\% and 93\% for SIIIDTG, $46 \%$ and $96 \%$ for SnIIIDTG, $83 \%$ and $98 \%$ for LCI, 55\% and $93 \%$ for Sacin, and $12 \%$ and $94 \%$ for Scond, respectively. Sensitivity for FEV1 was $34 \%$. In the $40(66 \%)$ children with CF and normal FEV1, SIIIDTG was abnormal in 18 (47\%) children (fig. 3), LCI was abnormal in $25(73 \%)$, Sacin in $13(41 \%)$ and Scond in one child.

\section{Double-tracer gas phase III slope and other lung function indices}

SIIIDTG was strongly associated with ventilation inhomogeneity measured by LCI and Sacin but not Scond (table 2 and fig. 4). 


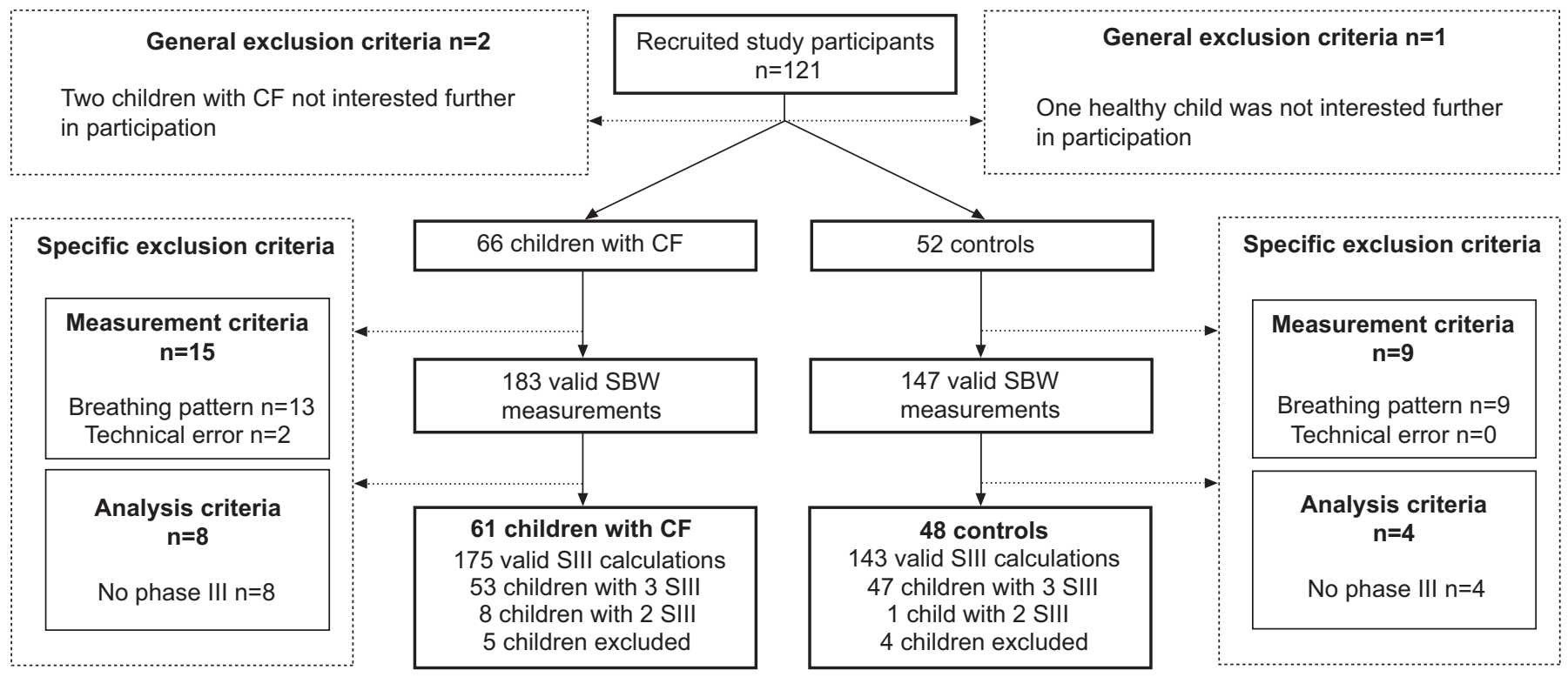

FIGURE 1. Success rate of double-tracer gas single-breath washout (SBW). Out of 121 children enrolled, 118 children (66 children with cystic fibrosis and 52 healthy children) performed 354 double-tracer gas SBW tests. Of these, 330 (93\%) conformed to measurement quality criteria of tidal phase IIII slope (SIII), and 318 (90\%) conformed to analyses quality criteria (online supplementary material). Technically acceptable SIII were available in 61 (92\%) children with CF and 48 (92\%) controls, and acceptable nitrogen multiple-breath washout indices were available in 54 (82\%) children with CF and 44 (85\%) controls. No severe adverse event occurred.

SIIIDTG decreased with increasing LCI $(r=-0.58)$ and Sacin $(\mathrm{r}=-0.58)$. SIIIDTG was weakly associated with airways obstruction measured by FEV1 ( $\mathrm{r}=0.26)$ (fig. 3) but not with FEF25-75\%.

\section{Quality control of double-tracer gas phase III slope}

Intra-test repeatability of SIIIDTG was acceptable and comparable between children with CF and control subjects. Comparing children with $\mathrm{CF}$ and controls, intra-test $\mathrm{CV}$ was $23.9 \%$ versus $18.5 \%$; the mean difference $(95 \% \mathrm{CI})$ of CV was $5.4(-3.8-14.5) \%$ $(\mathrm{p}=0.243)$. The mean intra-test variability of SIIIDTG (CV 22.5\%) was comparable to SnIIIDTG (CV 20.8\%) and mean difference $(95 \% \mathrm{CI})$ was $3.0(-2.7-8.8) \%$. The variability of SIIIDTG (CV) was higher than the CV of LCI $(7.4 \%)$, but was not associated with the primary outcome SIIIDTG, age or breathing pattern (online supplementary material). SIIIDTG data $(n=318)$ were reliably

\section{TABLE 1 Comparison between children with cystic fibrosis (CF) and healthy children}

\begin{tabular}{|c|c|c|c|c|}
\hline & CF subjects & Control subjects & Mean difference $(95 \% \mathrm{Cl})^{\#}$ & p-value \\
\hline Subjects & 61 & 48 & & \\
\hline Age yrs & $11.1 \pm 3.0$ & $11.8 \pm 2.9$ & $0.7(-0.4-1.8)$ & 0.224 \\
\hline Height z-score & $-0.57 \pm 1.20$ & $0.16 \pm 1.03$ & $0.72(0.23-1.21)$ & 0.004 \\
\hline Weight z-score & $-0.66 \pm 1.07$ & $-0.05 \pm 1.06$ & $0.61(0.16-1.10)$ & 0.009 \\
\hline SnlllDTG mg $\cdot \mathrm{mol}^{-1}$ & $-181.2 \pm 177.8$ & $-57.1 \pm 85.9$ & $124.1(68.7-179.4)$ & $<0.001$ \\
\hline MBNW n & 51 & 44 & & \\
\hline $\mathrm{LCl}$ & $12.1 \pm 3.5$ & $6.7 \pm 1.1$ & $5.4(4.3-6.5)$ & $<0.001$ \\
\hline Sacin & $0.23 \pm 0.16$ & $0.07 \pm 0.06$ & $0.16(0.11-0.21)$ & $<0.001$ \\
\hline Scond & $0.07 \pm 0.07$ & $0.04 \pm 0.06$ & $0.03(0.001-0.05)$ & 0.041 \\
\hline
\end{tabular}



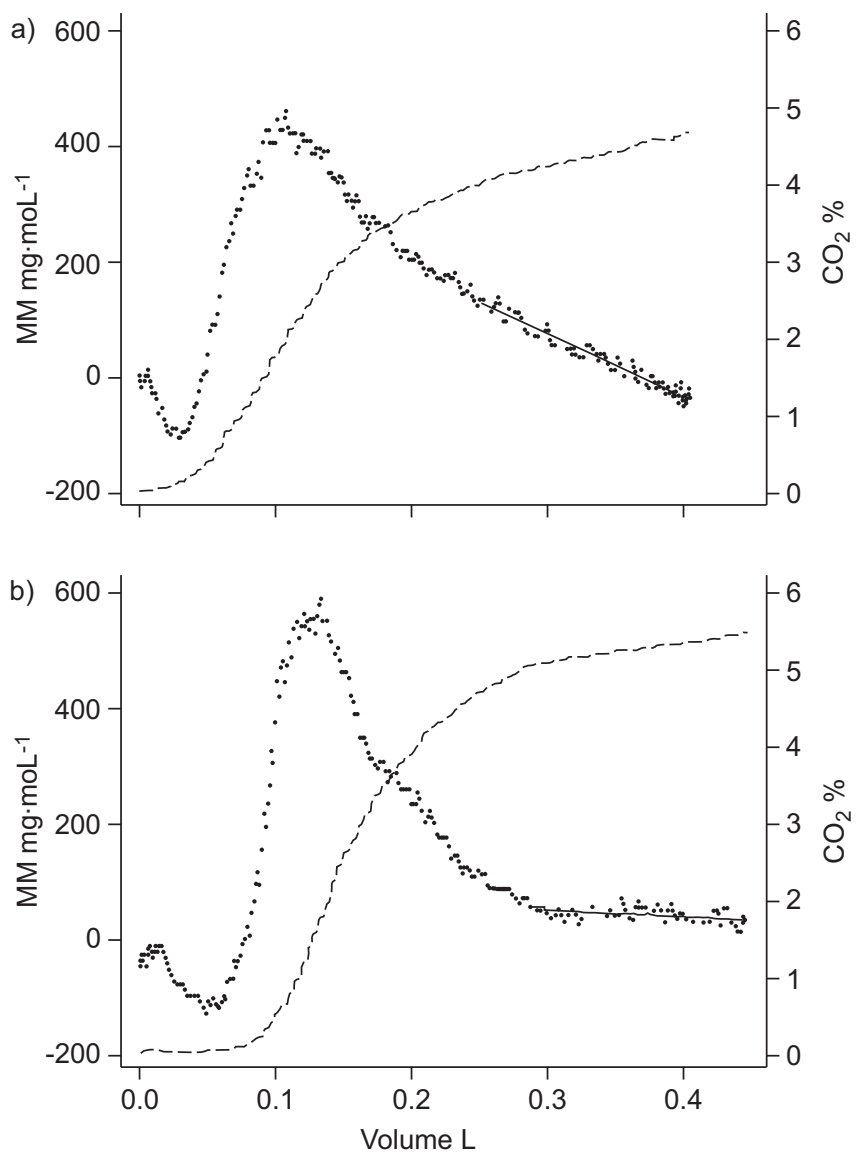

FIGURE 2. Typical expirograms of double-tracer gas single-breath washout (SBW). Double-tracer gas SBW giving molar mass (MM) samples (dots) and $\mathrm{CO}_{2}$ samples (dashes) plotted against expired volume in a) a male with cystic fibrosis and b) a control subject, both aged 9 yrs. Traditionally, SBW signals (e.g. $\mathrm{N}_{2}, \mathrm{SF}_{6}$ and $\mathrm{He}$ ) are plotted reversed with increasing tracer gas concentrations with increasing expired volume. We displayed the original $\mathrm{MM}$ curve with $\mathrm{SF}_{6}$ leading to a positive $\mathrm{MM}$ deflection and $\mathrm{He}$ leading to a negative $\mathrm{MM}$ deflection. $\mathrm{MM}>0$ reflects a greater $\mathrm{SF}_{6}$ than $\mathrm{He}$ contribution and, vice versa, $\mathrm{MM}<0$ reflects a greater He than $\mathrm{SF}_{6}$ contribution [16]. Investigators fitted the slope (solid line) according to the phase III of $\mathrm{MM}$ and $\mathrm{CO}_{2}$ between $65 \%$ and $95 \%$ of expired volume. Doubletracer SBW-derived tidal phase III slope (SIIIDTG) was a) $-591.6 \mathrm{mg} \cdot \mathrm{mol} \cdot \mathrm{L}^{-1}$ and b) $-65.8 \mathrm{mg} \cdot \mathrm{mol} \cdot \mathrm{L}^{-1}$.

calculated by two blinded, independent investigators (C. Abbas and F. Singer). Inter-observer variability of fitting quality (linear regression $R^{2}$ ) was negligible: mean $\pm S D$ linear regression fit $\mathrm{R}^{2}$ was $0.65 \pm 0.22$ and $0.63 \pm 0.23$. Inter-observer agreement for the outcome SIIIDTG was strong (intra-class correlation coefficient 0.92).

\section{DISCUSSION}

This new double-tracer gas tidal SBW detects early CF lung disease and is easy, fast and safe to perform. We obtained technically acceptable SIIIDTG in $92 \%$ of untrained school-aged children. To identify abnormal lung function in children with $\mathrm{CF}$, the tidal SBW was more sensitive than FEV1. Compared with indices derived from the much longer MBNW tests, SIIIDTG was comparably sensitive and specific Sacin, but less sensitive than the LCI. SIIIDTG was associated with Sacin and

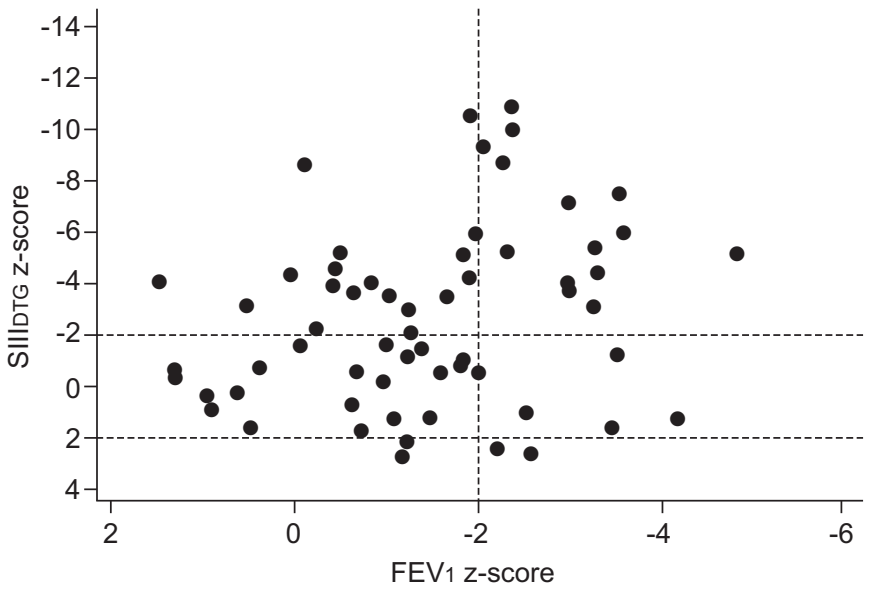

FIGURE 3. Association of double-tracer gas single-breath washout-derived phase III slopes (SIIIDTG) with airways obstruction. SIIIDTG were plotted against forced expiratory volume in $1 \mathrm{~s}\left(F E V_{1}\right)$ in 61 children with cystic fibrosis. $z$-scores for SIIIDTG and FEV 1 were calculated from the controls and reference data [22], respectively. Dashed lines reflect limits of abnormal lung function (two z-scores); the $y$-axis scaling is reversed.

global ventilation inhomogeneity (LCI), but not with Scond. SIIIDTG was repeatable within tests and reproducible between investigators. These data suggest that the double-tracer gas tidal SBW is a promising lung function test to assess ventilation inhomogeneity.

Classical VC SBW has previously been used to report steeper SIII for He than $\mathrm{SF}_{6}$ in patients with $\mathrm{CF}$ [12]. However, this result was deemed to have limited applicability to nonspecialised laboratories, due to the need for a mass spectrometer [15]. We now report a new SBW technique which dispenses with this need. Compared with the clinical applicability of previous MBW and VC SBW techniques, the advantages of the new tidal SBW demonstrated by our study lie within three major aspects: 1) an easy measurement protocol; 2) fast testing and analysis procedures; and 3) a set-up being available. This new doubletracer gas tidal SBW technique seems to have important basic requirements for clinical application. SIIIDTG was, by nature, obtained significantly faster and in a greater proportion of children compared with LCI or Sacin. This is important, as the number of feasible lung function tests in pre-school and early school-age is clearly limited by their test duration and protocol complexity. Interestingly, success rate and repeatability of tidal SBW in our study also compare preferably with previous VC SBW studies. In healthy children and adults, success rates of VC SBW ranged from $74 \%$ to $89 \%$, and intra-test variability of nitrogen SIII ranged between $13 \%$ and $24 \%$ [23, 24]. Previous MBW success rates ranged between $50 \%$ and $100 \%$ in children $[20,25,26]$. The need for complete tracer gas wash-in and leakfree washout with, for example, coughing or sighs, leading to MBW rejection, led to higher failure rates. However, LCI seems more repeatable than SIIIDTG $[6,25]$. This may be explained by measuring expired tracer gas volumes over a 10-times greater test duration as compared with the double-tracer gas tidal SBW. Further studies are needed to assess protocols reducing SIIIDTG variability that would also be applicable in children. 


\begin{tabular}{|c|c|c|c|}
\hline \multirow{2}{*}{$\begin{array}{l}\text { TABLE } 2 \\
\text { Lung function } \\
\text { z-score }\end{array}$} & \multicolumn{3}{|c|}{$\begin{array}{l}\text { Association of double-tracer gas single-breath } \\
\text { washout derived phase III slopes (SIIIDTG) with } \\
\text { other lung function indices }\end{array}$} \\
\hline & $\begin{array}{l}\text { Correlation } \\
\text { coefficient }\end{array}$ & $\begin{array}{c}\text { Crude } \\
\text { p-value }{ }^{\#}\end{array}$ & $\begin{array}{l}\text { Adjusted } \\
\text { p-value }\end{array}$ \\
\hline LCl & -0.58 & $<0.001$ & $<0.001$ \\
\hline Sacin & -0.58 & $<0.001$ & $<0.001$ \\
\hline Scond & -0.03 & 0.760 & 1.000 \\
\hline FEV $_{1}$ & 0.26 & 0.044 & 0.653 \\
\hline FEF $25-75 \%$ & 0.18 & 0.170 & 1.000 \\
\hline \multicolumn{4}{|c|}{$\begin{array}{l}\text { Association of SIIIDTG with indices from multiple-breath nitrogen washout in } 98 \\
\text { children ( } 54 \text { with cystic fibrosis) and spirometry in } 61 \text { children with cystic } \\
\text { fibrosis. Z-scores for SIIIDTG, lung clearance index (LCI), indices of conductive } \\
\text { (Scond) and acinar (Sacin) ventilation inhomogeneity were calculated from } \\
\text { controls, and for forced expiratory volume in } 1 \mathrm{~s}(\mathrm{FEV} 1) \text { and forced expiratory } \\
\text { flow at } 25-75 \% \text { of forced vital capacity (FEF } 25-75 \%) \text { are from reference data } \\
\text { [22]. Pearson correlation coefficients are given. }{ }^{*} \text { : crude p-values; }{ }^{\circ} \text { : Bonferroni } \\
\text { adjusted. }\end{array}$} \\
\hline
\end{tabular}

\section{Physiological and methodological considerations}

Increased overall ventilation inhomogeneity quantified by LCI or VC SBW using a single inert tracer gas may reflect increases in airway resistance, inhomogeneous changes in lung compliance, hyperinflation and alterations to lung structure. Regional ventilation inhomogeneity near the diffusion-convections fronts, which are specific for diffusivity of tracer gases, is thought to be the major determinant of the sloping phase III. The site along the airway tree primarily affected by ventilation inhomogeneity can be estimated using two approaches. One is the partitioning of MBW into Sacin and Scond [21,27]. The second method for localising inhomogeneity is by analysing tidal or VC SBW-derived SIII that are simultaneously obtained from two inert gases of different diffusivity, e.g. $\mathrm{SF}_{6}$ and $\mathrm{He}[12,28,29]$.
For light gases, such as $\mathrm{He}$, the diffusion-convection front is probably proximal but close to the acinus $[14,30]$. For the heavy gas $\mathrm{SF}_{6}$, this front may approximate to the entrance of the acinus and probably stretches into the proximal portion of the acinus. The exact anatomical correlates of these measures remain largely unknown.

This new tidal SBW method aggregates ventilation inhomogeneity of $\mathrm{He}$ and $\mathrm{SF}_{6}$. The reason these gases are aggregated is because all have simultaneous impact upon the molar mass signal. A steeply sloping SIIIDTG, which was significantly more common in children with CF compared with controls, means that significantly more $\mathrm{He}$ contributes to the phase III than $\mathrm{SF}_{6}$ ( $<-2$ z-scores) (figs 3 and 4) [16]. In contrast, steeply rising SIIIDTG ( $>2 \mathrm{z}$-scores) with significantly more $\mathrm{SF}_{6}$ contributing to the phase III than He was present in only four children. This suggests that airway pathology may be localised rather near the acinus entrance in the majority of children with CF. This assumption is supported by similar findings from VAN MUYLEM and BARAN [12] and the fact that steeper SIIIDTG was associated with increased Sacin. Increased He ventilation inhomogeneity compared with $\mathrm{SF}_{6}$ was also correlated to histological changes near the acinus entrance [29]. Recent magnetic resonance imaging studies visualised $\mathrm{He}$ ventilation inhomogeneity in children with CF [31]. However, we are not able to determine the exact location or nature of regional ventilation inhomogeneity leading to altered SIIIDTG in CF.

Tidal SBW performed from functional residual capacity is thought to be more reflective of peripheral airway function than that performed using a VC manoeuvre from residual volume [29]. Nevertheless, SIII may be influenced by breathing pattern, and raised volume protocols have arguably been regarded as being more robust [24, 32, 33]. However, shift in VC due to training effects introduces bias [24]. In younger subjects, a net multiplication of MBW derived SIII by $V \mathrm{~T}$ has been proposed [34]. In our study, SnIIIDTG did not improve intra-test variability or discriminatory capacity as compared with the simpler index SIIIDTG (online supplementary material). It remains to be
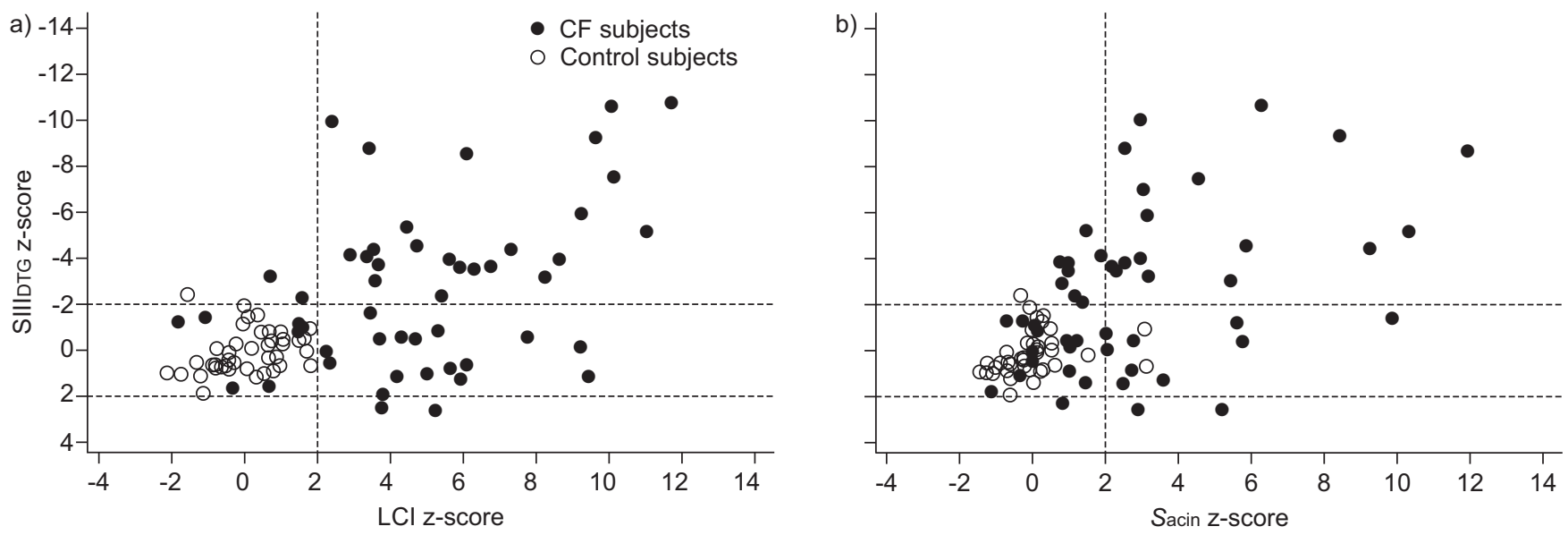

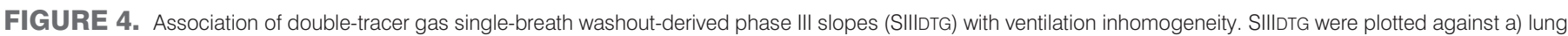

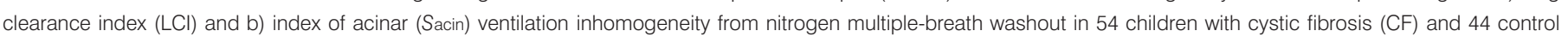

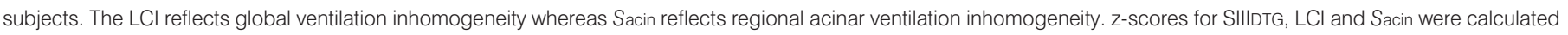

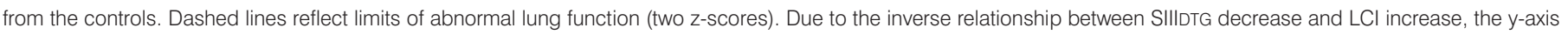
scaling is reversed. 
determined as to which approach to adjust for tidal breathing pattern post hoc is physiologically and clinically relevant.

This is the first study reporting SIII analysis derived from molar mass signals in lung disease. Compared with mass spectrometry signals, ultrasonic flowmeter derived molar mass does not require $\mathrm{SF}_{6}$ and $\mathrm{He}$ signal alignment and excellent agreement between molar mass and mass spectrometry signals has been shown [16]. SIIIDTG analysis quality $\left(R^{2}\right)$ and reproducibility between observers was good. Signal resolution is also higher, with a sampling rate of $200 \mathrm{~Hz}$ for the molar mass signal compared with current mass spectrometry setups at $33 \mathrm{~Hz}$.

\section{Implications for clinics and research}

This is the first study showing that tidal breath SIII analyses can identify abnormal lung function in CF using available equipment. A significant proportion of children with CF had normal FEV1 but abnormal indices of ventilation inhomogeneity. The weak correlation between FEV1 and SIIIDTG suggests that measures of distal ventilation distribution inhomogeneity (SIIIDTG) are sensitive in early CF lung disease, whereas measures of proximal airways obstruction (FEV1) are reliable in advanced CF lung disease. Assessing ventilation inhomogeneity in patients with normal FEV1 but who are at risk of small airway disease is considered particularly important as it may allow early intervention and prevent progress of disease [34]. In clinical routine, the double-tracer gas tidal SBW has three potential practical applications: 1) as a screening and monitoring tool in patients without respiratory symptoms or airway obstruction; 2) as an easy test for ventilation inhomogeneity in pre-school children; and 3) as an alternative test to MBW in primary care settings. Longitudinal change of He SIII as compared with $\mathrm{SF}_{6} \mathrm{SIII}$ seems very sensitive for early detection of bronchiolitis obliterans in patients at risk [11, 35]. Similar He, $\mathrm{SF}_{6}$, and $\mathrm{N}_{2} \mathrm{MBW}$ indices can detect increased ventilation inhomogeneity in patients with emphysema [36], primary ciliary dyskinesia [37], asthma [38, 39] and chronic obstructive pulmonary disease $[40,41]$.

This tidal SBW test may also be applicable for intervention trials in patients with small airway disease. While between-test variability of tidal SBW remains to be determined, time series measurements can be easily obtained and fluctuation over time of $\mathrm{He}$ and $\mathrm{SF}_{6} \mathrm{SIII}$ could be clinically relevant [11, 35]. Compared with $\mathrm{MBW}$, low exposure to dry tracer gas reduces the risk of triggering bronchial hyperreactivity. Lower consumption of inert gases and shorter test durations for tidal SBW as compared with MBW can reduce cost and decrease greenhouse gas emission, as $\mathrm{SF}_{6}$ is known to be a potent greenhouse gas.

\section{Conclusion}

This new double-tracer gas tidal SBW is a feasible, fast and safe test for detecting ventilation inhomogeneity in children with $\mathrm{CF}$ and healthy controls. The tidal SBW is performed during tidal breathing and outcomes are obtained within 4 min. SIIIDTG is associated with increased global and regional ventilation inhomogeneity near the acinar zone. The tidal SBW may be also promising for other patient groups with small airway disease. This study supports the applicability of the double-tracer gas tidal SBW as a sensitive and efficient lung function test to assess ventilation inhomogeneity.

\section{SUPPORT STATEMENT}

The work for this report was funded by the Swiss Society for Cystic Fibrosis, the Federal Department of Economic Affairs Switzerland, Commission for Technology and Innovation, Innovation Promotion Agency, unrestricted educational grant no. 11661.1 PFLS-LS, and the European Respiratory Society, Fellowship STRTF fellowship no. 812011. The funders had no role in study design, data collection and analysis, decision to publish, or preparation of the manuscript.

\section{STATEMENT OF INTEREST}

None declared.

\section{ACKNOWLEDGEMENTS}

The authors would like to thank all children and their families for their participation in the study. The authors would like to express their gratitude to P.M. Gustafsson (Dept of Paediatrics, Central Hospital, Skoevde, Sweden) and M. Roos (Dept of Paediatrics, University Hospital of Bern, Bern, Switzerland) for their valuable input, M. Schoeni and N. Regamey (Dept of Paediatrics, University Hospital of Bern) for their patient care, and S. Berger and A. Joeris (Dept of Paediatric Surgery, University Hospital of Bern) for their support in recruitment of controls. We express our gratitude to E. Kieninger, S. Yammine, O. Fuchs, E. Proietti, G. Wirz, M. Graf, S. Luescher, S. Schmid and C. Becher (Dept of Paediatrics, University Hospital of Bern) for their valuable work and support.

\section{REFERENCES}

1 Gustafsson PM, De Jong PA, Tiddens HA, et al. Multiple-breath inert gas washout and spirometry versus structural lung disease in cystic fibrosis. Thorax 2008; 63: 129-134.

2 Owens CM, Aurora P, Stanojevic S, et al. Lung clearance index and HRCT are complementary markers of lung abnormalities in young children with CF. Thorax 2011; 66: 481-488.

3 Ellemunter H, Fuchs SI, Unsinn KM, et al. Sensitivity of lung clearance index and chest computed tomography in early CF lung disease. Respir Med 2010; 104: 1834-1842.

4 Hall GL, Logie KM, Parsons F, et al. Air trapping on chest CT is associated with worse ventilation distribution in infants with cystic fibrosis diagnosed following newborn screening. PLoS One 2011; 6: e23932.

5 Hall GL, Stocks J. Intervention trials and ventilation distribution in mild cystic fibrosis lung disease: will it all come out in the wash? Eur Respir J 2011; 37: 757-759.

6 Amin R, Subbarao P, Jabar A, et al. Hypertonic saline improves the LCI in paediatric patients with CF with normal lung function. Thorax 2010; 65: 379-383.

7 Amin R, Subbarao P, Lou W, et al. The effect of dornase alfa on ventilation inhomogeneity in patients with cystic fibrosis. Eur Respir J 2011; 37: 806-812.

8 Belessis Y, Dixon B, Hawkins G, et al. Early cystic fibrosis lung disease detected by bronchoalveolar lavage and lung clearance index. Am J Respir Crit Care Med 2012; 185: 862-873.

9 Horsley A. Lung clearance index in the assessment of airways disease. Respir Med 2009; 103: 793-799.

10 Crawford AB, Makowska M, Paiva M, et al. Convection- and diffusion-dependent ventilation maldistribution in normal subjects. J Appl Physiol 1985; 59: 838-846.

11 Estenne M, Van Muylem A, Knoop C, et al. Detection of obliterative bronchiolitis after lung transplantation by indexes of ventilation distribution. Am J Respir Crit Care Med 2000; 162: 1047-1051.

12 Van Muylem A, Baran D. Overall and peripheral inhomogeneity of ventilation in patients with stable cystic fibrosis. Pediatr Pulmonol 2000; 30: 3-9. 
13 in 't Veen JC, Beekman AJ, Bel EH, et al. Recurrent exacerbations in severe asthma are associated with enhanced airway closure during stable episodes. Am J Respir Crit Care Med 2000; 161: 1902-1906.

14 Paiva M. Gas transport in the human lung. J Appl Physiol 1973; 35: 401-410.

15 Coates AL. Classical respiratory physiology - gone the way of the dinosaurs? Do we need a jurassic park? Pediatr Pulmonol 2000; 30: $1-2$.

16 Singer F, Stern G, Thamrin C, et al. Tidal volume single breath washout of two tracer gases - a practical and promising lung function test. PLoS One 2011; 6: e17588.

17 Beydon N, Davis SD, Lombardi E, et al. An official American Thoracic Society/European Respiratory Society statement: pulmonary function testing in preschool children. Am J Respir Crit Care Med 2007; 175: 1304-1345.

18 Singer F, Houltz B, Robinson PD, et al. A realistic validation study of a new nitrogen multiple-breath washout system. PLoS One 2012; 7: e36083.

19 Aurora P, Gustafsson P, Bush A, et al. Multiple breath inert gas washout as a measure of ventilation distribution in children with cystic fibrosis. Thorax 2004; 59: 1068-1073.

20 Gustafsson PM, Aurora P, Lindblad A. Evaluation of ventilation maldistribution as an early indicator of lung disease in children with cystic fibrosis. Eur Respir J 2003; 22: 972-979.

21 Verbanck S, Schuermans D, Vincken W. Inflammation and airway function in the lung periphery of patients with stable asthma. J Allergy Clin Immunol 2010; 125: 611-616.

22 Stanojevic S, Wade A, Cole TJ, et al. Spirometry centile charts for young Caucasian children: the Asthma UK Collaborative Initiative. Am J Respir Crit Care Med 2009; 180: 547-552.

23 Teculescu DB, Pham QT, Hannhart B, et al. Computerized singlebreath nitrogen washout in children: variability and reproducibility. Clin Physiol 1987; 7: 247-259.

24 Teculescu DB, Rebstock E, Caillier I, et al. Variability of the computerized single-breath nitrogen washout test in healthy adults. Results from a field survey in a French rural area. Clin Physiol 1993; 13: 35-50.

25 Aurora P, Bush A, Gustafsson P, et al. Multiple-breath washout as a marker of lung disease in preschool children with cystic fibrosis. Am J Respir Crit Care Med 2005; 171: 249-256.

26 Fuchs SI, Ellemunter H, Eder J, et al. Feasibility and variability of measuring the lung clearance index in a multi-center setting. Pediatr Pulmonol 2012; 47: 649-657.
27 Gustafsson PM. Peripheral airway involvement in CF and asthma compared by inert gas washout. Pediatr Pulmonol 2007; 42: 168-176.

28 Gustafsson PM, Ljungberg HK, Kjellman B. Peripheral airway involvement in asthma assessed by single-breath $\mathrm{SF}_{6}$ and $\mathrm{He}$ washout. Eur Respir J 2003; 21: 1033-1039.

29 Van Muylem A, De Vuyst P, Yernault JC, et al. Inert gas singlebreath washout and structural alteration of respiratory bronchioles. Am Rev Respir Dis 1992; 146: 1167-1172.

30 Lacquet LM, Van Muylem A. He and SF6 single-breath expiration curves. Comparison with the paiva-engel model. Bull Eur Physiopathol Respir 1982; 18: 239-246.

31 McMahon CJ, Dodd JD, Hill C, et al. Hyperpolarized ${ }^{3}$ helium magnetic resonance ventilation imaging of the lung in cystic fibrosis: comparison with high resolution CT and spirometry. Eur Radiol 2006; 16: 2483-2490.

32 Crawford AB, Cotton DJ, Paiva M, et al. Effect of lung volume on ventilation distribution. J Appl Physiol 1989; 66: 2502-2510.

33 Crawford AB, Makowska M, Engel LA. Effect of tidal volume on ventilation maldistribution. Respir Physiol 1986; 66: 11-25.

34 Robinson PD, Goldman MD, Gustafsson PM. Inert gas washout theoretical background and clinical utility in respiratory disease. Respiration 2009; 78: 339-355.

35 Van Muylem A, Antoine M, Yernault JC, et al. Inert gas singlebreath washout after heart-lung transplantation. Am J Respir Crit Care Med 1995; 152: 947-952.

36 von Nieding G, Lollgen $H$, Smidt U, et al. Simultaneous washout of helium and sulfur hexafluoride in healthy subjects and patients with chronic bronchitis, bronchial asthma, and emphysema. Am Rev Respir Dis 1977; 116: 649-660.

37 Green K, Buchvald FF, Marthin JK, et al. Ventilation inhomogeneity in children with primary ciliary dyskinesia. Thorax 2012; 67: 49-53.

38 Farah CS, King GG, Brown NJ, et al. The role of the small airways in the clinical expression of asthma in adults. J Allergy Clin Immunol 2012; 129: 381-387.

39 Verbanck S, Schuermans D, Paiva M, et al. Nonreversible conductive airway ventilation heterogeneity in mild asthma. J Appl Physiol 2003; 94: 1380-1386.

40 Verbanck S, Schuermans D, Paiva M, et al. Small airway function improvement after smoking cessation in smokers without airway obstruction. Am J Respir Crit Care Med 2006; 174: 853-857.

41 Verbanck S, Schuermans D, Meysman M, et al. Noninvasive assessment of airway alterations in smokers: the small airways revisited. Am J Respir Crit Care Med 2004; 170: 414-419. 